\title{
The Development and Innovation of Chinese Guzheng Playing Techniques
}

\author{
Mingming Qu \\ Xi'an Conservatory of Music, Xi'an, Shaanxi Province, China \\ 2481533218@qq.com
}

Keywords: Guzheng, playing, techniques, development and innovation

\begin{abstract}
Guzheng is one of Chinese traditional Musical Instruments, in schools and local characteristics are distinct. On the one hand, contemporary inherited the art of the traditional Guzheng playing the Guzheng, on the other hand, to achieve innovation basis on the inheritance.At the same time, also has carried on the reference to some other instruments, is of great help to play the Guzheng, and implements the Guzheng playing technique development and innovation. In this paper, the representative of Guzheng playing techniques are analyzed, and pointed out the Guzheng playing techniques in that part of the inheritance and innovation in the development of parts.
\end{abstract}

\section{Introduction}

Guzheng is one of the common plucked instruments, is also one of the oldest plucked-string instrument. Plucked instruments and other instruments of the difference are the main characteristic of the instrument is based on "points" as the main characteristics of play when that feature "granularity". Music is, however, boundless change, need millions of melody to express ideas in music, just use "dot" is difficult to express music world what happens in a graceful melody and music scene [1]. So music requires constant development, and constantly innovation, make a musical instrument by the form of different can play all kinds of beautiful moving melody, in order to make the play the Guzheng art can be able to better development in social life, makes Guzheng playing along in the process of continuously perfect, clever people will "dot" and "line" clever unifies in together, make the Guzheng forms more diversification, performance ability is stronger, the performance scene is more abundant. This article mainly analyzes the contemporary form played Guzheng playing techniques and induction, and summarizes the characteristic of its.

\section{The origin historical of Guzheng}

Guzheng is an ancient ethnic Musical Instruments, during the warring states period in the "Qin", $\mathrm{Gu}$ zheng is a "real" qin is, so is the name of "qin zheng". About two views are named, the first argument is a splitting, and another is for pronunciation possessed the name.

Guzheng is only five strings, initially formed as a result of the limitation of string Guzheng narrow range, in the warring states period, people in order to increase the width of the range, also increased the seven strings, a total of 12 root. In the later development process has been used in the form of the necks, until after 1300 years of the Tang and Song dynasties and add a root to 13 strings, although only increase a piece of string, but with music professional judging standard, increase the use value is greater than ever before [1]. Unprecedented in the history of China's development in the Tang dynasty is a brilliant, all areas during this period has been an unprecedented development, the same Guzheng has also been an unprecedented development in this period, by improving their necks formed ten three-stringed Guzheng. In the meantime, there are both twelve string zheng and 13 strings zither, both exist, formation and low. Popular in court is called gagaku necks zheng zheng, popular in the folk is called ten three-stringed zheng, vulgar music Guzheng following the Tang dynasty andSong dynasty, the Guzheng music system. When the Tang dynasty, zheng is widely used, ts 'en-ts 'an's 
poem is used to practice the Guzheng [1]. Tang dynasty Guzheng performers also emerge in endlessly, such as the famous Guzheng performers Hao Su. In the period of sui, get further development in this field, with 14 string zither and fifteen strings zither. Popular during the Ming dynasty, the palace is fifteen strings, string is used, but due to play skilled problem and zheng music performance ability, the most widely popular in folk or ten three-stringed zheng. Because ten three-stringed zheng in the long process of development is comparatively mature technology, can give full play to the space is larger. In the late Qing dynasty in the early of the republic of China, there are 16 strings zither, along with the development of The Times, so far, in the modern zheng is dominant in the 21st string, and was identified as affordable koto strings. There are a few 25 strings zither and 26 strings zither, but less common in the play.

\section{The development of Guzheng playing techniques and the existing problems}

The ancient people think of little finger as playing Guzheng "ban", think to use when playing the piano when using the little finger is not allowed, this habit gradually evolved into the traditional, this tradition until today, people also influence the practice Guzheng techniques, people playing Guzheng pinky sit still "bench", so the little finger always play a supporting role, such as in the use of relatively fine fingering in Guzheng - shake refers to, if the thumb as the point at which the shaking, little finger can only as a strong point to pile, to balance the plucked string fingers, even if sometimes used, is only plucked a few times, therefore, little finger has been as "idle" [2]. You always have a doubt, little playing western instruments or some Chinese instruments will play the role of and other fingers of the same size, but why can't in Guzheng playing. Contrast before the ring finger, had rapidly refers to the sequence in the undeveloped, ring finger when playing some chord may use, but also is idle most of the time, but in the coming of the above techniques, in the use of the finger is increased greatly, and other fingers are equally important, especially in the so-called "wheel" four fingers fingering after being used, the role of the ring finger is even more highlighted.

The collective spirit of cooperation consciousness is not good. One-to-one teaching is now Guzheng teaching in classroom teaching mode. Actually this kind of teaching way also exist some shortcomings, it will spend as much time and effort, the teacher while teachers' income is high, but can appear the phenomenon of high investment and low yield can, on the other hand will seriously affect the communication between students, let them lose many could have cooperation opportunities. It is not hard to see, one to one teaching mode on the personality development of students, even the formation of students' healthy personality is bad, so often there will be a personal performance level is high, but they don't know how to work together with others, not cooperation skill, harmony and balance of parts in the ensemble are sure not to live [2]. It can cause some students prefer to further solo is not willing to cooperate with other students, collective consciousness is indifference.

Mentality problem. In today's highly commercialized society, some teaching institutions and teachers in order to cater to the utilitarian mentality, that ignored the Guzheng learning rule and do not take the art quality.The pure pursue high repertoire lets the student in the short term furiously practicing a song to deal with two. The distinction of individual institutions only tests a tune do not take an examination of etude, body of sight-reading phenomenon common occurrence, also can easily get the certificate, but actually these students don't have to reach the qualified level, the distinction is the summary of a certain period of learning to learners of Chinese zither, this no doubt has lost its original meaning [2].

\section{Guzheng technique characteristics and inheritance}

Guzheng is a kind of instrument with a long history, has been widely spread in China. According to the geographical features, Guzheng playing techniques can be divided into north and south two factions;According to the style characteristics, Guzheng playing techniques are divided into nine types. In the heritage and development of Guzheng playing techniques in the process of the folk customs, culture, politics, geography, under the influence of such factors as different local colour and rhyme has been derived and innovation [3]. 
The technique characteristics of traditional Guzheng. The characteristics of the traditional Guzheng mainly playing techniques for the "left hand press, the right hand to play zheng", the development and reform of Guzheng has a very long history [3]. Through "right hand department play" refers to the right hand thumb and index finger and ring finger to pluck the strings to make a sound, at the same time to control the notes and rhythm. "Left hand department according to the" refers to by the movements of the left by the string, to the application of right hand polished, change the right hand play rhythm and strength of notes.

1) The right hand characteristics. In the process of playing the Guzheng, elastic string is the most important way of traditional hand techniques, strings are generally in the right side of the play, is also the Guzheng voice of the most basic tasks. The traditional Guzheng techniques, right hand technique is mainly with their thumb and forefinger, middle finger, ring finger to control the strength of the sound and rhythm [3]. Single tone techniques in playing the Guzheng is one of the most common techniques, and fingers to play method is single tone techniques the main content. Right hand techniques and some common, including li tone techniques, continuous sound techniques and polyphony techniques, etc.

2) The left hand characteristics. In the process of playing the Guzheng, traditional left hand techniques is the most main technique according to the string, using finger pressure, in the top left of the Guzheng to control chords. Techniques is the main task of the left hand act the role of sound, as the name implies, the function of the left hand is on the adornment of the melody, embellish beauty of tone color, has a great effect on the formation of a Guzheng rhythm characteristics [4]. The playing technique of the left hand still has a lot of kinds, such as the glide, vibrato and point the sound and so on. Enable each finger to play in the play of their use.Known as the king of the western instruments piano fingering is symmetrical, that is to say, fingering is his left hand and right hand fingering difference is little, that is to say, the piano is trained hands.For Guzheng, any practitioners in just started learning when I learn right hand, left hand just do some songs, knead, slide, quiver of practice, just as an accompaniment to use left hand.

The inheritance of traditional Guzheng. Although the modern Chinese zither playing techniques have been relatively rapid development, has obtained certain achievements, but in the process of playing the Guzheng, still occupied the main position is one of the traditional Guzheng playing techniques [4]. The important play method about Guzheng, Guzheng or "quiver, note, Yin, according to the" traditional playing techniques, such as the skill in playing the Guzheng reveal a unique charm to let a person for a long time to praise. At present, there are many new clever tunes, also increased a lot of new complex way of playing, but did not adopt new methods of tuning in Guzheng instruments or a new playing techniques. In Guzheng performance, the most important way or in the "chatter, note, according to Yin" and other different playing style is given priority.

\section{The innovation of the Guzheng playing techniques}

In the process of modern Chinese zither playing, the most important innovation is to realize the liberation of the left hand, left hand to be able to break out of the limit of jean code on the left side of the involved in the guitar code on the right side of the play [5]. Both hands to play Guzheng techniques first appeared in 1935, the most typical representative is "general", applied to hold zheng technique. But these methods at the time did not receive adequate attention and communication; it was not until later got further development.

Guzheng playing techniques innovation. With the rapid development of China's economic and social, traditional Guzheng playing techniques also got certain development, Guzheng education obtained the popularization, and the professional education. Under this background, the traditional Guzheng playing techniques and continuous development and innovation, revealed a more sound, the characteristics of heavy and complicated, in developing the most outstanding performance is improved the traditional playing techniques [5]. In the traditional Guzheng playing techniques, is an important geographical factors, with the advancement of cultural fusion, in the process of modern zheng music creation must to innovate traditional Guzheng performance techniques. First of all, have 
a positive improvement, the traditional Guzheng playing techniques is one of the most important specification in the process of playing posture, hand type, etc. In carried on the inheritance to the traditional Guzheng playing techniques in the process of the most common way is KouChuanXinShou, in this way to teach playing techniques, such as a song. This way of inheritance has led to the playing position, he can't get effective norms such as the shape of the hand.

1) Specification of Guzheng playing position. In the process of playing the Guzheng, a major position including seated with vertical two kinds. Seated refers to in the process of playing the Guzheng need to sit in the chair half or less than half of the position, if in the process of sitting occupies the entire chair, will cause the body center of gravity after the case, produce adverse effect to play the Guzheng. In the process of using sitting to play, two feet left foot are at ease in the former, through three ways to control the body [6]. On the bench to choose according to the actual situation of the players, in the process of parallel to Guzheng music yards around the waist with players sat down. If the bench to play too high will cause the inconvenient, if stool is too low will cause players arm fatigue;In the process of sitting, the body of the players to keep a certain distance with jean body side panel, if close to the rim will affect the attitude of the players.

2) Guzheng play specification that you touch. Guzheng tone under the influence of Guzheng itself on the one hand, on the other hand, is affected by the performance method. In the process of playing the Guzheng adopt different ways to play, voices are different. In the process of playing the Guzheng, usually employing methods mainly include the two plays, clip to play two playing technique [6]. Mention playing method refers to the fingers in the process of playing in the direction of the elastic string oblique bottom to top to play, using this way to play the sound in terms of sound quality is relatively pure, clean, more crisp, bright in tone. Clip play method refers to the fingers in the process of playing oblique to the above the bottom of the direction of play to play, using this way to play the sound in terms of sound quality is full, in terms of timbre is relatively thick.

Other ethnic instruments techniques Guzheng. On Guzheng playing techniques in the process of development and innovation, to achieve a certain carrier and Guzheng instruments characteristics in combination with each other, so as to realize the development of Guzheng playing techniques.

1) Use of onomatopoeia sexual techniques. Guzheng has a relatively rich in traditional Chinese Musical Instruments of music expression [7]. Right hand in the guitar code on the right side of the string to play, to make music, left hand on the harp strings of code on the left side of the "Yin", "according to", "rubbing", etc. At present, many composers in the midpoint of the Guzheng music expressive force, realize the expansion of the music expression, in the process of exploring new techniques to achieve the hands playing position liberation, created the visual impact and auditory impact force are very powerful new techniques, simulate all kinds of sound.

2) The west playing techniques of Guzheng. Zheng play not only inherit the development of Guzheng music culture, but also affected by the western music. Absorb and draw lessons from the part of the western Musical Instruments playing techniques [7]. Traditional Guzheng playing techniques in the process of playing, just use middle finger finger thumb to play. After the western way of playing, has realized the five fingers bold application in Guzheng performance. In addition, the left hand in the process of playing the Guzheng, breakthrough the limitation of the harp yards left, also participated in the code on the right side of the strings, playing the rich to implements the Guzheng music performance.

\section{Conclusion}

In Guzheng playing techniques in the process of the heritage and development, on the one hand, under the influence of Guzheng playing techniques inherent law, on the other hand, under the influence of the external environment, such as social culture. With the constant communion of culture and promoting the further development of China Guzheng playing techniques. Under this background, 
China Guzheng playing technique to realize the continuous development and innovation, in the future, there will be more new performance techniques.

\section{References}

[1] L.L.Liang, the development of contemporary Chinese zither techniques, Journal of Xi 'an college of music, 2011, vol.(2),pp.57-60.

[2] J.T. Li, The contemporary style of Guzheng art diversity and play the technology standardization, Chinese music, 2011,vol.(1), pp.21-24.

[3] P.D. Sheng, Guzheng refers to the sequence technique introduction to fast, People's music, 2012, vol.(12), pp.44-48.

[4] Y.D. Zhao, Guzheng playing techniques of rheological and its problems in today's teaching, Journal of Wuhan music institute, 2012, vol.(11), pp.13-17.

[5] X.X Fang, Guzheng posture rhythm of playing and teaching, Division of Hunan journal of van, 2009, vol. (02), pp.98-101.

[6] L.T.Chang, The adult of Guzheng teaching my opinion, The public art, 2009, vol.(3), pp.12-15.

[7] S. M. Yang, Mysteries and discuss Guzheng teaching in university, Journal of Yangtze university (social science edition). 2008, vol.(6),pp.52-55. 